\title{
Pervasive Wireless-Sensor-Networks for Home Healthcare Need Automatic Reasoning
}

\author{
Davide Merico, Alessandra Mileo and Roberto Bisiani \\ NOMADIS Lab., University of Milano-Bicocca, \\ viale Sarca, 336/14, I-20126 Milan MI, Italy. \\ e-mail: \{davide.merico, alessandra.mileo, roberto.bisiani\}@nomadis.unimib.it
}

\begin{abstract}
This paper deals with the problems of using pervasive wireless sensor networks to monitor people that live in their homes and have no critical chronic condition. The rich set of sensors that can be used and their sheer number make it quite complex to interpret the data: in the paper we argue that it is possible and very useful to add a reasoning component to the architecture. We also delve into the various open research questions that must be solved in order to build really effective home health-monitoring systems.
\end{abstract}

Keywords-Wireless Sensor Networks, Home Healthcare, Reasoning.

\section{INTRODUCTION}

$\mathrm{W}$ HILE life-expectancy continues to grow, at least in some countries, the inequality in life expectancies and Healthy Life Years (HLYs) [1] remains large. This means that many people live longer but in a state in which chronic conditions substantially cripple their quality of life and their capability of living a useful life. Furthermore, this inequality has a negative impact on society welfare costs.

Tele-monitoring of critical conditions (telemedicine) is becoming very common because of lower and lower costs of equipment and the savings that can be achieved. Telemedicine, though, does not always improve HLY's because it enters the picture when a person has already one or more critical chronic conditions. On the contrary, pervasive monitoring in the home (which has been proposed in research projects and, in a somewhat reduced form, in commercial products) has the potential of prolonging the HLYs period by pinpointing potentially critical conditions before they arise.

Many "independent living" systems focus on capturing medical emergencies. This can be useful but it is not the most effective way to improve the quality of life of elderly people or to lower medical costs. A more useful goal is to prevent situations that can cause drastic changes for the worse of the quality of living, e.g. falls, constant weight loss, etc. We believe that this can be achieved with pervasive and continuous monitoring of an elderly in his home environment supported by sophisticated processing of the indicators computed from the monitoring.

It has been shown that, for example, hip fractures continue to generate significant costs throughout the firstyear period after discharge. Costs associated with the treatment of hip-fracture patients are about three times greater than those resulting from the treatment of age and residence-matched controls without a fracture [2]. As a matter of fact, causes of falls in elderly adults are quite diverse, the largest culprits being: environment-related $(31 \%)$, gait/balance disorders $(17 \%)$ and diziness/vertigo (13\%) [3]. Identifying risk factors can substantially lower the probability of a fall. It is therefore very advantageous both for the patient's quality of life and for the minimization of the costs to devise systems that are able to warn caregivers and patients that action has to be taken well before a fall happens.

The architecture of an effective independent-living support system should include:

- A Wireless Sensor Network (WSN), composed of infrastructural nodes equipped with several sensors and a wearable monitoring device, used for gathering data about the user and the environment.

- A reasoning component able of performing three main reasoning tasks: (i) continuous contextualization of physical, mental and social state of a person, (ii) prediction of possibly risky situations and (iii) identification of plausible causes for the worsening of a person's health.

We believe research should be mainly focusing on:

Unobtrusiveness The monitoring system should not affect the lifestyle and habits of the person being monitored. All data should be unobtrusively captured by the WSN and then processed without any user intervention.

User-friendliness The elderly may have problems in handling complex multiple devices. The monitoring system should allow interactions with the person through a very simple interface, e.g. a touch sensitive display and simplified graphic interface.

Context-awareness Gathering complete information about the context in which the user lives is a decisive point for home healthcare systems. The use of a reasoning component that does not only rely on static user-specific needs, but that continuously analyzes the evolving state of the patient and of the environment, simplifies the situation assessment process. The aggregation and the interpretation of different kinds of information from heterogeneous sources (such as light, position, movement, localization, load cells) enhances reliability and accuracy of context interpretation, since considering heterogeneous sources of information helps in compensating errors and incompleteness of data.

Reactivity The reasoning process should run not only periodically but also according to specific triggers such as emergency situations, user input, system feedbacks. By 


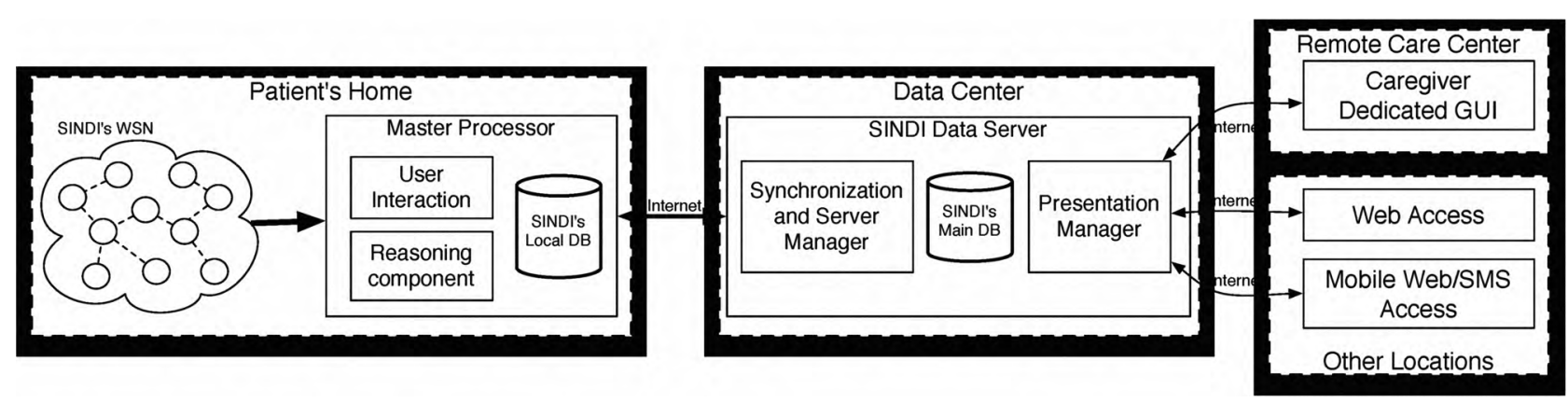

Fig. 1. The Overall Architecture of SINDI.

adding appropriate logical constraints to the WSN and to the reasoning components, emergencies could also be contextualized in almost real-time.

Other important requirements like: (i) technological and medical soundness, (ii) user-centrality, (iii) adaptivity and (iv) affordability should also be addressed.

In the remainder of this paper we will use the description of a system we have designed and implemented, called Secure and INDependent LIving (SINDI) [4], in order to illustrate the key problems in pervasive health monitoring.

\section{System OverviEW}

In order to address the concerns expressed in Section I we have designed and developed a system (called SINDI) that has the following components.

The SINDI's WSN used for gathering data about the user and his or her environment.

The Master Processor used for storing and processing data. This processor also handles all the connections with the WSN and the Data Server and it has storage, processing power and main memory capabilities in the ballpark of an average $\mathrm{PC}$.

The SINDI Data Server used to store and manage the data of several homes.

The SINDI Clients used by caregivers to access patient's information stored on the SINDI Data Server.

The overall architecture of the SINDI system is depicted in Figure 1.

In the remaining part of this paper we will focus on the most significative components of the SINDI system: the WSN, the Master Processor and the Reasoning Component.

\section{ThE WSN OF SINDI}

Wireless Sensor Networks (WSNs) [5] consist of nodes that are capable of interacting with the environment by sensing or controlling physical parameters. This kind of networks are the enabling technology for acquiring all possible information about the context in which the user lives.

The WSN we use in SINDI collects environmental data about light, temperature, humidity, opening and closing of doors and windows. Several data about user's movement, location and weight (using load cells under a bed or an armchair) are also collected. The architecture of SINDI's WSN is composed by:
- a base node in every zone, always active and connected to household power, mostly used for network coordination but also with its own sensing capabilities;

- environmental nodes (battery powered) for sensing the environmental data or capturing particular events;

- a wearable monitoring device;

- a gateway node connected to the master processor.

Both the base nodes and the environmental nodes can sense at least: temperature (10-40 degrees with one degree precision), humidity (low-normal-high) and light changes that are meaningful to people. Furthermore, they use Received Signal Strength Indication (RSSI), ultrasonic- and infrared-sensor data for localization and proximity.

The base node integrates a power supply and a small rechargeable battery for power outages. The wearable monitoring device is used for the user's localization and also includes several sensors (accelerometers, gyroscopes and magnetometers) for movement analysis. The gateway node is the coordinator of the networks and it is used by the master processor for gathering data from the WSN.

The network is organized hierarchically. The environment in which the user lives is divided into zones and every zone is controlled by one base node. Moreover, every zone can be divided into several sensing areas where one or more environmental nodes operate. The wearable node can move from zone to zone without loss of connectivity. The master processor manages the entire network applying topology-control mechanisms and routing algorithms.

Several middleware environments that provide routing, data aggregation, and communication services have been designed in order to optimize and limit resource consumption. For instance, middlewares like [6] or [7] are good examples of generic environments based on message exchange mechanisms optimized for homogeneous sensor networks. These environments, however, do not provide enough flexibility to manage heterogeneous sensors and do not meet all our requirements. We developed a middleware that provides the following functionalities: (i) dynamic configuration of the nodes and simple data aggregation (ii) communication, routing, power control and synchronization (iii) positioning, localization and tracking.

The key research issues in this kind of network are energy management, network bandwidth and data filtering.

Although the network is deployed in a home there is a definite advantage in having most of the sensor nodes 


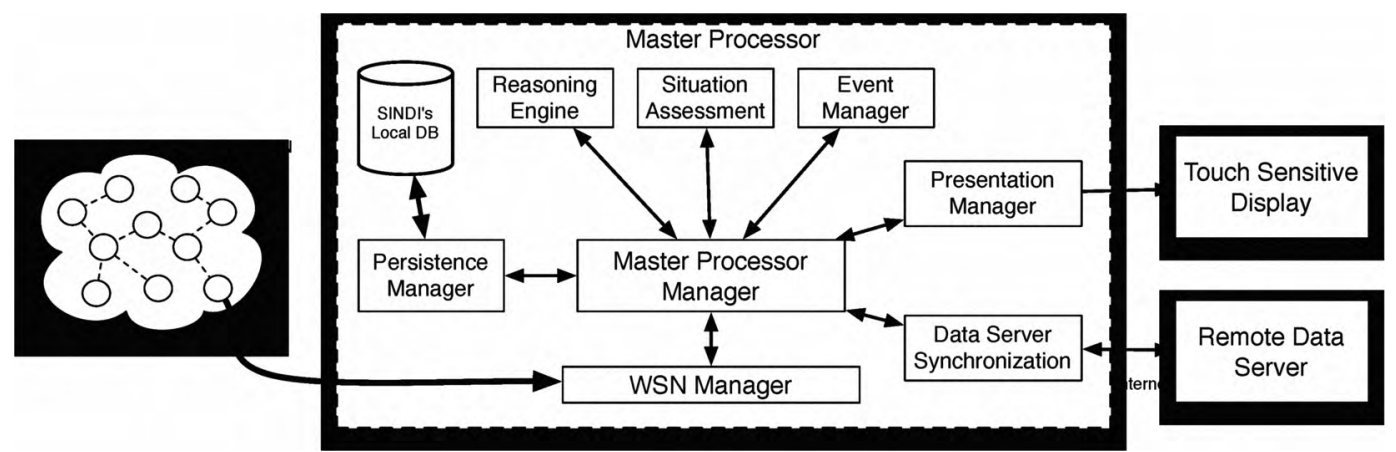

Fig. 2. A Detailed View of the Master Processor Components.

battery operated in order to obtain low installation costs and unobtrusiveness. While most sensors do not need to be read very often, localization needs frequent interactions. The key is to exploit all the context knowledge in order to minimize the number of measurements that need to be taken. For example, the presence or the arrival of a user in a room can be inferred from context data saving RSSI measurements. Another issue has to do with the tradeoff between energy consumption and the capability of the network to react to emergencies. A clever management of sleep time is required in order to always have the possibility for the network as a whole to react to emergencies.

Bandwidth and Medium Access Control are another serious problem. The most common MAC protocol, IEEE 802.15 .4 , does a good job if contention is low but adds quite a bit of overhead if too many nodes are eager to transmit. Here again the application has to cleverly exploit the context knowledge in order to "throttle-back" the nodes.

Timely collecting data from sensor nodes is not enough because such data are noisy, incomplete and potentially inconsistent. Only the most sophisticated data filtering techniques are good enough for some of the sensors that are used in these systems, e.g. accelerometers and gyroscopes on a human body, and one often has to resort to complex data filtering and fusion techniques, e.g. particle filters, in order to improve accuracy.

\section{The Master Processor}

As we have seen, the Master Processor is the gateway of the WSN and it is used for storing and processing data. A detailed view of the Master Processor components can be found in Figure 2. It includes the following subcomponents.

Master Processor Manager This component is used for handling the communication between all the other modules.

Situation Assessment This component is used for processing and aggregating the raw user and environmental data gathered with the WSN in order to find more detailed information about the context. In this way, detailed information about the user localization are obtained combining together the inertial and RSSI data using a fusion algorithm based on particle filters. The raw user movement data are filtered using algorithms based on decision trees and artificial neural networks in order to obtain data with higher quality. Algorithms for filtering raw ultrasonic and infrared data are also used.

Reasoning Engine This component allows a constant monitoring of the user health using the contextual data processed by Situation Assessment, further refining them and also using user-specific medical knowledge (see section V). Event Manager This component is used in order to guarantee quick reactions when critical situations and emergencies arise.

WSN Manager This component is used to correctly acquire all the environmental and user data generated by the WSN. It is also used for the configuration of the WSN.

Persistence Manager and Local Database All the system data are stored in a local database using the Persistence Manager component.

Presentation Manager It is used to manage the user interactions with GUIs that are specifically designed for elderly people.

Data Server Synchronization It synchronizes the local and remote database interacting with the remote SINDI's Data Server.

A detailed view of the Master Processor components can be found in Figure 2.

This is probably the easier part of the system since most of the design issues are solved by existing technologies. The only difficult issues are raised by the user interface that needs to be very different from usual interfaces because it must be used mainly by people with little or no familiarity with computers.

\section{INTELLIGENT MONITORING: A LOGICAL PERSPECTIVE}

We believe that future systems should go beyond data collection to focus on (i) assisting caregivers in understanding health evolution, (ii) enhancing independence and autonomy of monitored patients. We refer to an intelligent monitoring system as a monitoring system that is able to reason about gathered data and support decisions. Most of the pervasive systems for healthcare proposed so far use a probabilistic approach to behaviour analysis and activity recognition aimed at enhancing autonomy [8]. These approaches are sometimes coupled with logic-based planning techniques.

When we talk about intelligent monitoring, though, we 
refer to a different (potentially complementary) view of artificial intelligence applied to home healthcare. In our view, expressive knowledge representation and reasoning techniques are needed to analyse the context and to understand health evolution. This can be done by applying automated reasoning to a combination of different pieces of knowledge (common-sense, medical, context-dependent), rather than dealing with predefined plans and goals to be achieved.

The idea is that of using non-monotonic logical reasoning to summarize and correlate sensor data in a consistent interpretation of the context in which the person lives in terms of clinical profile, environment, movements, and to predict possible evolutions of the person's health. The logical framework of Answer Set Programming (ASP) [9] is well suited to deal with such a complex knowledge representation and reasoning task, in that it overcomes most of the limitation of previous logic programming systems such as Prolog. Compared to pure statistical approaches, logic inference based on ASP is highly expressive and computationally more performant because it can deal with first-order representations, which are much richer than the propositional ones characterizing probabilistic inference. Furthermore, ASP can deal with incomplete information and commonsense reasoning using defaults. Cardinality and weight constraints together with program optimization techniques can also be used to model different degrees of uncertainty [10], [11], [12].

In the SINDI system, we combine Wireless Sensor Networks technologies with a logic-based representation of knowledge. The reasoning component of SINDI uses Clingo as the ASP reasoning engine ${ }^{1}$. In our implementation, three main reasoning tasks are performed by the system: Contextualization is referred to as the correlation between sensor data and physical, mental and social state of the person; this task is based on common-sense reasoning and domain knowledge and it provides a qualitative evaluation of crucial aspects of daily living (movement, quality of sleep, clinical setting).

Prevention combines results of the contextualization task with medical knowledge in order to identify potentially risky situations before the emergencies arise.

Local Diagnosis is referred to as (i) the identification of possible causes for the worsening of a person's health (provided as a result of the contextualization task) and (ii) the indication of the clinical and context-dependent conditions producing a given risk assessment as a result of the prevention task.

Results of the reasoning process can also be used to give feedbacks and suggestions to both the caregivers and the patients in form of prompts, alerts, warnings, and so on.

There are several open issues concerning logic-based artificial intelligence applied to home healthcare, among which we mention:

- correct encoding and integration of the appropriate medical knowledge;

- successful combination of probabilistic and logic-based approaches for modelling and reasoning which can enhance

\footnotetext{
${ }^{1}$ http://potassco.sourceforge.net/
}

adaptivity and user-centrality;

- careful study of which feedback should be provided by the system, when it is needed and how it should be provided; - usability and access to technology by the elderly.

\section{CONCLusions}

In conclusion we can see that there is a large difference between the usual telemedicine systems and this new breed of pervasive monitoring systems. First of all, data collection raises many performance and energy issues. The network must be available $24 / 7$ and continuously adapt to the monitoring context, run unattended for long periods of time and be installed by unskilled personnel. Secondly, sensor data must be conditioned and filtered by sophisticated techniques that can deal with complex sensor models, e.g. particle filters. Last but not least, data has to be interpreted by a component that is able to draw from a knowledge database and make complex inferences.

More details and a preliminary evaluation of such a system can be found in [4].

\section{REFERENCES}

[1] C. Jagger et al., "Inequalities in healthy life years in the 25 countries of the european union in 2005: a cross-national metaregression analysis," The Lancet, vol. 372, no. 9656, pp. 21242131, 2008

[2] P. Haentjens, G. Lamraski, and S. Boonen, "Costs and consequences of hip fracture occurrence in old age: An economic perspective," Disability Rehabilitation, vol. 27, no. 18-19, pp. 1129-1141, 2005.

[3] L. Z. Rubenstein, "Falls in older people: Epidemiology, risk factors and strategies for prevention," Age and Ageing, vol. 3552, pp. ii37-ii41, 2006.

[4] D. Merico, A. Mileo, S. Pinardi, and R. Bisiani, "A Logical Approach to Home Healthcare with Intelligent Sensor-Network Support," The Computer Journal, p. bxn074, 2009.

[5] I. F. Akyildiz, W. Su, Y. Sankarasubramaniam, and E. Cayirci, "A survey on sensor networks," IEEE Communications Magazine, vol. 40, no. 8, pp. 102-114, 2002.

[6] Eduardo Souto, Germano Guimarães, Glauco Vasconcelos, Mardoqueu Vieira, Nelson Rosa, and Carlos Ferraz, "A messageoriented middleware for sensor networks," in Proceedings of $M P A C$ 2004, Toronto, Ontario, Canada, 18-22 October 2004, pp. 127-134, ACM, New York, NY, USA.

[7] P. Costa, G.P. Picco, and S. Rossetto, "Publish-subscribe on sensor networks: a semi-probabilistic approach," in Proceedings of MASS 2005, Washington, DC, 7-9 November 2005, pp. 332342, IEEE Computer Society, Los Alamitos, CA, USA.

[8] Karen Zita Haigh and Holly Yanco, "Automation as caregiver: A survey of issues and technologies," in Proceedings of the AAAI-02 Workshop "Automation as Caregiver", 2002, pp. 3953, AAAI Technical Report WS-02-02.

[9] M. Gelfond and V. Lifschitz, "The stable model semantics for logic programming," in Proceedings of ICLP 88, Seattle, Washington, 15-18 August 1988, pp. 1070-1080, MIT Press, Massachussets, USA.

[10] P. Simons, I. Niemelä, and T. Soininen, "Extending and implementing the stable model semantics," Artificial Intelligence Journal, vol. 138, no. 1-2, pp. 181-234, 2002.

[11] N. Leone, G. Pfeifer, W. Faber, T. Eiter, G. Gottlob, S. Perri, and F. Scarcello, "The dlv system for knowledge representation and reasoning," ACM Transactions on Computational Logic, vol. 7 , no. 3, pp. 499-562, 2006.

[12] M. Gebser, B. Kaufmann, A. Neumann, and T. Schaub, "Conflict-driven answer set solving," in Proceedings of IJCAI 2007, Hyderabad, India, 6-12 January 2007, pp. 386-392, AAAI Press, Massachussets, USA. 